\title{
OPEN Amorphous Ge-Bi-Se Thin Films: A Mass Spectrometric Study
}

\author{
Ravi Mawale ${ }^{1 *}$, Govinda Mandal ${ }^{2}$, Marek Bouška ${ }^{1}$, Jan Gutwirth ${ }^{1}$, Pankaj Lochan Bora ${ }^{2}$, \\ Virginie Nazabal ${ }^{1,3}$, Josef Havel ${ }^{2}$ \& Petr Němec $^{1 *}$
}

The Ge-Bi-Se thin films of varied compositions (Ge content 0-32.1 at. \%, Bi content 0-45.7 at. \%, Se content 54.3-67.9 at. \%) have been prepared by rf magnetron (co)-sputtering technique. The present study was undertaken in order to investigate the clusters generated during the interaction of laser pulses with $\mathrm{Ge}-\mathrm{Bi}$-Se thin films using laser ablation time-of-flight mass spectrometry. The stoichiometry of the clusters was determined in order to understand the individual species present in the plasma plume. Laser ablation of Ge-Bi-Se thin films accompanied by ionization produces about 20 positively and/or negatively charged unary, binary and ternary $\left(\mathrm{Ge}_{x}{ }^{+}, \mathrm{Bi}_{y}{ }^{+}, \mathrm{Se}_{z}{ }^{+l-}, \mathrm{Ge}_{x} \mathrm{Se}_{z}{ }^{+l-}, \mathrm{Bi}_{y} \mathrm{Se}_{z}{ }^{+l-}\right.$ and $\mathrm{Ge}_{x} \mathrm{Bi}_{y} \mathrm{Se}_{z}{ }^{-}$) clusters. Furthermore, we performed the laser ablation experiments of Ge:Bi:Se elemental mixtures and the results were compared with laser ablation time-of-flight mass spectrometry analysis of thin films. Moreover, to understand the geometry of the generated clusters, we calculated structures of some selected binary and ternary clusters using density functional theory. The generated clusters and their calculated possible geometries can give important structural information, as well as help to understand the processes present in the plasma processes exploited for thin films deposition.

Amorphous chalcogenides have received great attention due to their unique properties and a broad range of applications. Chalcogenide compounds have emerged as multipurpose material and have been used for various applications for solid-state devices ${ }^{1}$. Due to their specific properties, chalcogenide glasses and their amorphous thin films are applied in photonics ${ }^{2}$, solar cells ${ }^{3}$, phase change memories ${ }^{4,5}$, optical fibers ${ }^{6}$, etc.

Among many chalcogenide glasses, selenium-based ones are significant because of their wide transparency window in the infrared spectral region, strong non-linear optical properties, high refractive index, and good mechanical, thermal and chemical characteristics ${ }^{2,7-9}$. For example, especially germanium selenides show good mechanical properties, such as hardness, adhesion, low internal stress, and water resistance ${ }^{10,11}$. In fact, Ge-Se glasses are thoroughly studied from the point of view of glass-forming region, their structural features, and physical properties ${ }^{11,12}$. Physical properties of binary or ternary chalcogenide glasses based on the Ge-Se system can be further adjusted by adding other elements. Specifically, the incorporation of Bi to the chalcogenide Ge-Se-based glasses and thin films leads to a very rare effect, namely n-type conductivity due to carrier reversal change, while the addition of other elements of the same group $(\mathrm{As}, \mathrm{Sb})$ does not ${ }^{13-16}$. The observed $\mathrm{n}$-type conductivity may be related to the increased metallic properties of bismuth as compared to As and Sb; hence an addition of bismuth may control, in an effective way, electrical/optical properties of Ge-Se-based glasses and thin films. As a consequence, amorphous Ge-Bi-Se chalcogenide thin films are investigated to study their optical and electrical properties $^{17-19}$, structure $^{17,20,21}$, etc.

Various deposition techniques are used for the fabrication of thin films from chalcogenide glasses. These are, for example, pulsed laser deposition, thermal evaporation, chemical vapor deposition, radio-frequency magnetron sputtering, etc ${ }^{22}$. Of special interest is $\mathrm{rf}$ magnetron co-sputtering, which may produce thin films with different stoichiometries by varying only the electrical power applied to the individual sputtering targets. Indeed, co-sputtering is a cost-effective process for the investigation of compositional dependencies of thin films' characteristics. One of co-sputtering's key features is that it enables the fabrication of amorphous thin films with compositions outside the glass-forming region of corresponding bulk counterparts ${ }^{23,24}$.

As mentioned above, different characterization techniques have been employed to examine Ge-Bi-Se glasses and amorphous thin films in order to understand their characteristics. It has been shown that irradiation of amorphous chalcogenides - in their bulk as well as thin film forms - with a pulsed laser produces many clusters

${ }^{1}$ Department of Graphic Arts and Photophysics, Faculty of Chemical Technology, University of Pardubice, Studentská 573, 53210, Pardubice, Czech Republic. ²Department of Chemistry, Faculty of Science, Masaryk University, Kamenice 5/A14, 625 00, Brno, Czech Republic. ${ }^{3}$ Institut des Sciences Chimiques de Rennes, UMR-CNRS 6226, Equipe Verres et Céramiques, Université de Rennes 1, 35042, Rennes, France. *email: mawale.ravi@gmail.com; petr.nemec@upce.cz 


\begin{tabular}{|l|l|l|l|l|l|}
\hline \multirow{2}{*}{$\begin{array}{l}\text { Sample } \\
\text { name }\end{array}$} & \multicolumn{2}{|l|}{ rf power (W) } & \multicolumn{3}{l|}{ Chemical composition (at. \%) } \\
\cline { 2 - 7 } & $\mathrm{GeSe}_{2}$ & $\mathrm{Bi}_{2} \boldsymbol{S e}_{3}$ & $\mathbf{G e}$ & $\boldsymbol{B i}$ & $\mathbf{S e}$ \\
\hline $\mathrm{A}$ & 20 & 0 & 32.1 & 0 & 67.9 \\
\hline $\mathrm{B}$ & 29 & 6 & 27.6 & 7.0 & 65.4 \\
\hline $\mathrm{C}$ & 25 & 10 & 20.5 & 16.5 & 63.0 \\
\hline $\mathrm{D}$ & 21 & 14 & 12.4 & 26.5 & 61.1 \\
\hline $\mathrm{E}$ & 15 & 20 & 6.1 & 35.7 & 58.2 \\
\hline $\mathrm{F}$ & 0 & 20 & 0 & 45.7 & 54.3 \\
\hline
\end{tabular}

Table 1. Thin films identification, $r f$ sputtering power used on both sputtering targets, and chemical composition of the co-sputtered Ge-Bi-Se thin films determined using EDS technique $( \pm 1$ at. \%).

via laser ablation processes, as confirmed through time-of-flight mass spectrometry. This technique can be used to elucidate the structural fragments present in the plasma generated during the interaction of high energy laser pulses with chalcogenide glasses ${ }^{25-27}$. However, mass spectrometric study of Ge-Bi-Se materials has not yet been reported, and therefore it is carried out here.

The aim of this work is to investigate Ge-Bi-Se amorphous chalcogenide thin films fabricated via rf magnetron co-sputtering. The fabricated films were characterized in detail using laser ablation time-of-flight mass spectrometry (LA TOFMS) analysis. The stoichiometry of the species generated from parafilm coated and non-coated amorphous $\mathrm{Ge}-\mathrm{Bi}-\mathrm{Se}$ thin films was determined and the results were discussed. Further, we also compared the clusters generated from $\mathrm{Ge}$-Bi-Se layers with those originating in mixtures of $\mathrm{Ge}$, $\mathrm{Bi}$, and Se elements. Finally, for selected binary and ternary species, their structure was calculated using density functional theory (DFT).

\section{Results and Discussion}

The thin films of different compositions, labelled A-F in Table 1, prepared via magnetron co-sputtering were characterized by scanning electron microscopy; the images show that the morphology of the layers is of good quality with smooth surfaces, and marginal presence of inhomogeneities. The elemental composition of the fabricated thin films was obtained by EDS at different spots of the films and averaged (Table 1). The EDS data indicate that single cathode sputtering (at $20 \mathrm{~W}$ ) gives thin films with a composition in line with the $\mathrm{GeSe}_{2}$ and $\mathrm{Bi}_{2} \mathrm{Se}_{3}$ targets used. In case of co-sputtering, by using rf power 15-29 and 6-20 W for $\mathrm{GeSe}_{2}$ and $\mathrm{Bi}_{2} \mathrm{Se}_{3}$ targets respectively, the co-sputtered films cover a wide compositional range: $6.1-27.6$ at. $\%$ of $\mathrm{Ge}, 7.0-35.7$ at. \% of Bi, and 58.2-65.4 at. \% of Se. XRD patterns revealed that all the deposited films (except $\mathrm{Bi}_{2} \mathrm{Se}_{3}$ ) are amorphous. The thickness of the thin films are within the range $660-1010 \mathrm{~nm}( \pm 2 \mathrm{~nm})$.

The co-sputtered Ge-Bi-Se thin films, as well as elemental mixtures of $\mathrm{Ge}, \mathrm{Bi}$, and $\mathrm{Se}$, were examined via LA TOFMS in both positive and negative ion modes. During LA accompanied by ionization, the interaction of laser pulses with the materials under investigation generated many positively and negatively charged ions as well as clusters. The peaks with intensities higher than $1 \mathrm{mV}$ are considered as relevant and their stoichiometries were determined by comparing experimental and theoretical isotopic patterns.

The effect of laser power on the generation of clusters was studied by systematically increasing the laser energy (90-180 a.u.). Through this procedure, the threshold energy at which ionization starts was determined for each sample in both positive and negative ion modes. For thin films, the threshold energy was identified between 120130 a.u., while for the elemental mixtures of $\mathrm{Ge}, \mathrm{Bi}$, and Se, it is $90-100$ a.u. No signals were obtained after $\mathrm{m} / \mathrm{z}$ 1200 , therefore, only mass spectra up to this $\mathrm{m} / \mathrm{z}$ value are shown in the figures. It was noticed that the intensity of the peaks rises with increasing laser energy; however their mass resolution is decreased. Therefore, the 'optimal' mass spectra with sufficiently high mass resolution and the largest number of clusters were obtained for each sample in both modes of measurement. In addition, the parafilm coated thin films, as well as elemental mixtures dispersed in parafilm solution, were also examined and the results compared.

Ge-Bi-Se thin films. Positive ion mode. Co-sputtered amorphous Ge-Bi-Se thin films were investigated via laser ablation TOFMS. Mass spectra with sufficient resolution and maximum number of detected species were obtained at laser energy between 140-150 a.u.

LA of $\mathrm{GeSe}_{2}$ binary thin (sample A) film generated many unary and binary species (Fig. 1). The lowest mass positively charged ion obtained from the $\mathrm{GeSe}_{2}$ thin film is $\mathrm{Ge}^{+}$, which is partially overlapped with $\mathrm{Se}^{+}$. Several other binary $\mathrm{Ge}_{\mathrm{x}} \mathrm{Se}_{\mathrm{z}}{ }^{+}$clusters are also detected. In addition, other unary $\mathrm{Se}_{2}{ }^{+}, \mathrm{Ge}_{8}{ }^{+}$and some oxygenated species were identified. The oxygenated species might be sourced from the partial oxidation of the surface of the thin films. The results obtained from thin films A are in agreement with our previously published results from the binary Ge-Se system ${ }^{27}$. However, due to the somewhat lower resolution of the instrument used in this work, the stoichiometry of some peaks could not be determined. For the sample $\mathrm{F}\left(\mathrm{Bi}_{2} \mathrm{Se}_{3}\right)$, the lowest mass ion detected was $\mathrm{Se}^{+}$, and many binary $\mathrm{Bi}_{\mathrm{y}} \mathrm{Se}_{\mathrm{z}}{ }^{+}$as well as unary $\mathrm{Bi}_{\mathrm{y}}{ }^{+}$clusters were detected, of which $\mathrm{Bi}^{+}$ion has the highest intensity.

The measured LA TOFMS data for ternary Ge-Bi-Se thin films (samples B, C, D, and E) are shown in Fig. 2. The stoichiometry of clusters obtained in all these samples is not significantly different except their intensities. The lowest mass clusters obtained in sample $\mathrm{B}$ and $\mathrm{C}$ is $\mathrm{Ge}^{+}$, which is overlapped with $\mathrm{Se}^{+}$cation. Several series of other clusters were detected. For example, sample $\mathrm{E}$ shows $\mathrm{Bi}_{y}^{+}(y=1-5), \mathrm{Bi}_{y} \mathrm{Se}^{+}(y=1-4), \mathrm{Bi}_{3} \mathrm{Se}_{z}^{+}(z=1-4)$, i.e. 


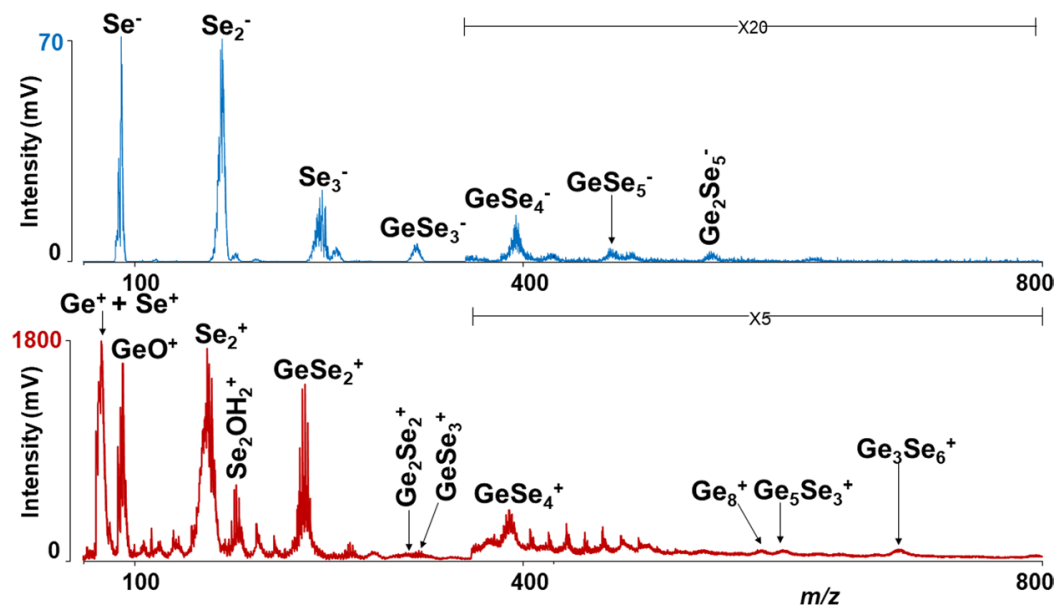

Figure 1. Mass spectra of sample A. Conditions: positive (red)/negative (blue) ion modes, laser energy 145 and 140 a.u. respectively.

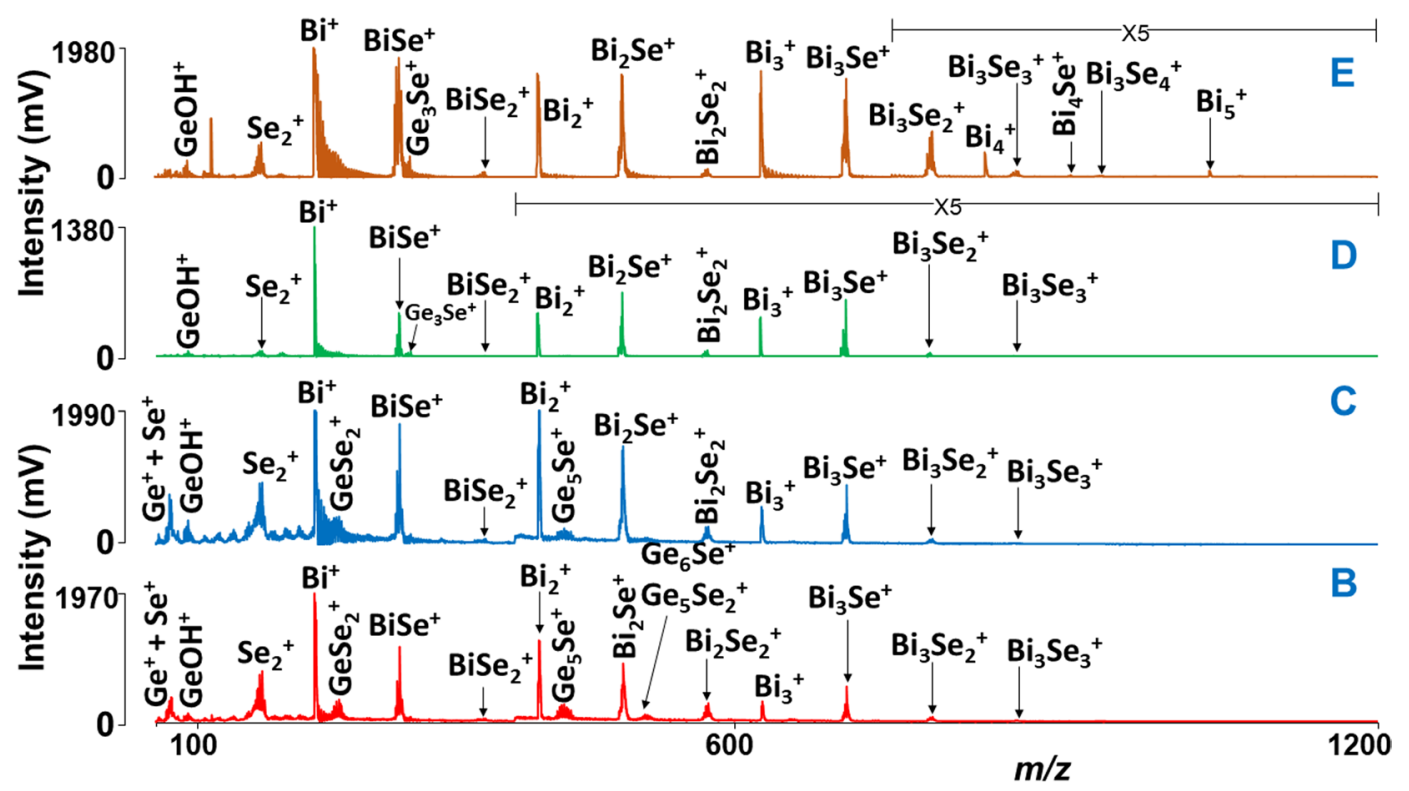

Figure 2. Mass spectra of Ge-Bi-Se thin films. Conditions: positive ion mode, laser energy 150 a.u.

series of clusters with increasing number of $\mathrm{Bi}$ or Se atoms. The $\mathrm{Bi}^{+}$ion is the highest intensity peak observed in all the samples, followed by $\mathrm{BiSe}^{+}$. It was observed that the $\mathrm{Bi}_{y}{ }^{+}$or $\mathrm{Bi}_{y} \mathrm{Se}^{+}$clusters dominate the mass spectra with their intensity and number of clusters as compared to the $\mathrm{Ge}^{+}$or $\mathrm{Ge}_{x} \mathrm{Se}_{z}{ }^{+}$ones. This seems to be related to the chemical composition of thin films. From ternary Ge-Bi-Se layers, sample B has lowest Bi and highest Ge content, while for sample $\mathrm{E}$ it is the opposite; therefore as one goes from sample $\mathrm{B}$ to $\mathrm{E}$, the number of $\mathrm{Ge}^{+}$or $\mathrm{Ge}_{x} \mathrm{Se}_{z}^{+}$species and their intensities are reduced. Logically, the number of $\mathrm{Bi}_{y}{ }^{+}$or $\mathrm{Bi}_{y} \mathrm{Se}^{+}$species and their intensities show an opposite trend. It is worth noting that no binary $\mathrm{Ge}_{x} \mathrm{Bi}_{y}{ }^{+}$or ternary $\mathrm{Ge}_{x} \mathrm{Bi}_{y} \mathrm{Se}_{z}{ }^{+}$clusters were detected in positive ion mode.

In addition, parafilm coated thin films were examined and the mass spectra were recorded. However, no significant difference in the mass spectra was observed as compared to the mass spectra of non-coated thin films; therefore, the mass spectra are not shown here. The intensity of the peaks in the mass spectra of coated thin films is lower as compared to non-coated thin films.

Negative ion mode. In negative ion mode, the threshold energy was determined to be between 120-130 a.u. for all the samples. The 'richest' mass spectra with sufficient mass resolution and with a maximum number of clusters were obtained at laser energy $140-155$ a.u. like in positive ion mode. LA of binary $\mathrm{GeSe}_{2}$ thin film generated $\mathrm{Se}_{z}^{-}(z=1-3), \mathrm{GeSe}_{z}{ }^{-}(z=3-5)$, and $\mathrm{Ge}_{2} \mathrm{Se}_{5}{ }^{-}$species (Fig. 1, blue spectrum). In the case of the $\mathrm{Bi}_{2} \mathrm{Se}_{3}$ thin films, only negatively charged $\mathrm{Se}_{z}{ }^{-}(z=1-4)$ clusters were identified. When samples $\mathrm{B}, \mathrm{C}, \mathrm{D}, \mathrm{E}$ were investigated by LA TOFMS, no significant differences between individual mass spectra were observed with respect to the number 

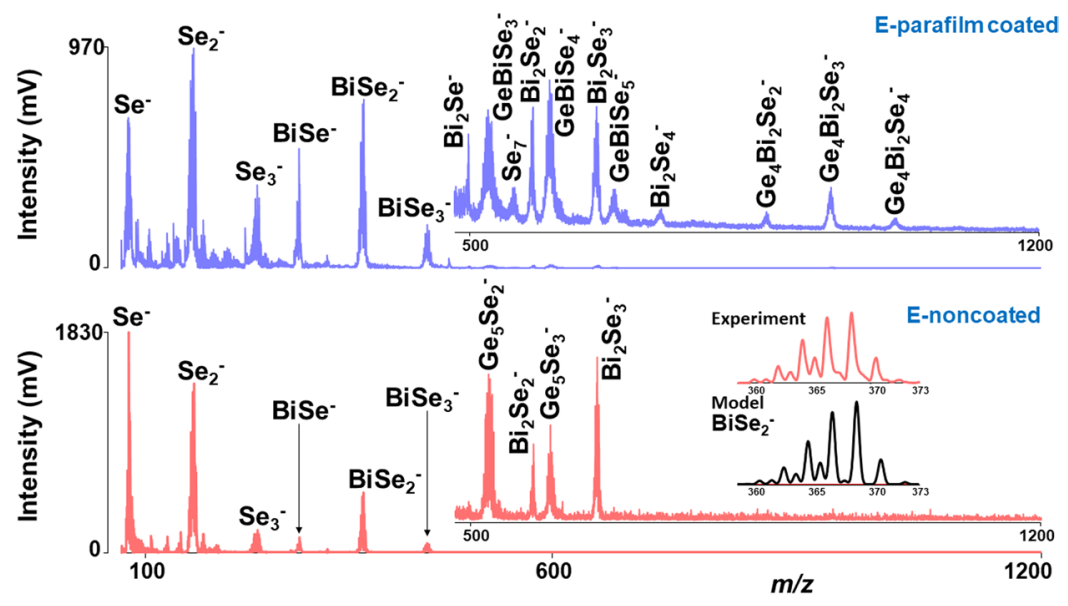

Figure 3. Mass spectra of parafilm coated and non-coated thin film E. Conditions: negative ion mode, laser energy 155 a.u. Part of mass spectra in the range $m / z 490-1200$ is magnified.

and stoichiometry of the detected species. As an example, the mass spectrum of sample $\mathrm{E}$ is given in Fig. 3. Series of unary $\mathrm{Se}_{z}{ }^{-}(z=1-3)$ and binary $\mathrm{BiSe}_{z}{ }^{-}(\mathrm{z}=1-3)$ species were detected. In addition, $\mathrm{Bi}_{2} \mathrm{Se}_{2}{ }^{-}$and $\mathrm{Bi}_{2} \mathrm{Se}_{3}{ }^{-}$and a few $\mathrm{Ge}_{x} \mathrm{Se}_{z}{ }^{-}$clusters were identified. A comparison of the experimental and theoretical isotopic patterns of the selected cluster $\left(\mathrm{BiSe}_{2}{ }^{-}\right)$, which shows their good agreement, is given in the inset of Fig. 3. It was observed that in negative ion mode, the selenium clusters are more intensive as compared to most of $\mathrm{Bi}_{y} \mathrm{Se}_{z}{ }^{-}$clusters. Also, negatively charged $\mathrm{Ge}$ or Bi species were not detected. This might be due to the electron affinity value of selenium which is higher than both $\mathrm{Ge}$ and $\mathrm{Bi}$ (electron affinity values of $\mathrm{Se}, \mathrm{Ge}$, and $\mathrm{Bi}$ are 2.02, 1.23, and 0.94) ${ }^{28}$.

When mass spectra were recorded from parafilm coated Ge-Bi-Se thin films, new higher mass ternary clusters $\left(\mathrm{GeBiSe}_{3}^{-}, \mathrm{GeBiSe}_{4}^{-}, \mathrm{GeBiSe}_{5}{ }^{-}, \mathrm{Ge}_{4} \mathrm{Bi}_{2} \mathrm{Se}_{2}{ }^{-}, \mathrm{Ge}_{4} \mathrm{Bi}_{2} \mathrm{Se}_{3}{ }^{-}, \mathrm{Ge}_{4} \mathrm{Bi}_{2} \mathrm{Se}_{4}{ }^{-}\right)$with an increasing number of selenium atoms were detected. In addition, new species such as $\mathrm{Bi}_{2} \mathrm{Se}^{-}, \mathrm{Se}_{7}{ }^{-}$and $\mathrm{Bi}_{2} \mathrm{Se}_{4}{ }^{-}$were generated. The comparison of mass spectra from parafilm coated and non-coated thin films is given in Fig. 3. As in positive ion mode, it was observed that the intensity of the mass spectra peaks in negative ion mode is lower for parafilm coated thin films than for uncoated ones. This indicates that higher laser energy is required for the ionization of the ions for the coated thin films, which is also supported by the fact that higher threshold energy was observed for coated thin films.

Laser ablation of Ge:Bi:Se elemental mixtures. Later, we deal with the LA TOFMS of elemental mixtures of Ge, $\mathrm{Bi}$, and Se with different molar ratios (1:1:1, 1:1:5, 1:5:1, and 5:1:1). To record the mass spectra from elemental mixtures, a similar methodology and experimental conditions as for the thin films were employed. The mass spectra were recorded at varied laser energy between 70-180 a.u.; threshold energy was determined to be between 90-100 a.u. In positive ion mode, mass spectra obtained from all the Ge:Bi:Se precursors show the formation of many unary, binary and a few ternary clusters. A comparison of mass spectra obtained from all the Ge:Bi:Se elemental mixture precursors are given in Fig. 4. Typically, unary $\mathrm{Ge}^{+}, \mathrm{Bi}^{+}$ions, and $\mathrm{Se}_{\mathrm{z}}^{+}(\mathrm{z}=2-8)$ species were observed. Most of the binary clusters correspond to the $\mathrm{Bi}_{\mathrm{y}} \mathrm{Se}_{\mathrm{z}}{ }^{+}$general formula with an increasing number of $\mathrm{Bi}$ and $\mathrm{Se}$ atoms. For example, these are $\mathrm{BiSe}_{\mathrm{z}}{ }^{+}(\mathrm{z}=1-7), \mathrm{Bi}_{2} \mathrm{Se}_{\mathrm{z}}{ }^{+}(\mathrm{z}=2-5)$, and $\mathrm{Bi}_{3} \mathrm{Se}_{\mathrm{z}}{ }^{+}(\mathrm{z}=1-6)$. Moreover, $\mathrm{BiGe}_{3}{ }^{+}$ and $\mathrm{BiGe}_{4}{ }^{+}$binary species were detected. Particularly, for the LA of $\mathrm{Ge}: \mathrm{Bi}: \mathrm{Se}(1: 1: 1)$ the highest intensity peak corresponds to the $\mathrm{Se}_{5}{ }^{+}$cluster, while several selenium oxygenated species were also identified. In the mass spectra of Ge:Bi:Se (1:1:5), where selenium is in excess, all the above-mentioned clusters were found. Logically, due to excess selenium, the peaks assigned to the selenium clusters are of higher intensity than the rest of the other peaks in the mass spectra. Nevertheless, the intensity of the peak connected with the $\mathrm{Bi}^{+}$ion is the highest among all the peaks. This observation can be due to a lower value of ionization energy reported for $\mathrm{Bi}^{+}$compared with $\mathrm{Se}^{+}(16.7 \mathrm{vs} \text {. } 21.1 \mathrm{eV})^{29}$. In the case of the mass spectra recorded from the (1:5:1) precursor with an excess of bismuth, $\mathrm{Bi}_{\mathrm{y}}{ }^{+}(\mathrm{y}=1-3)$ ion/clusters were revealed. Few ternary hydrogenated $\mathrm{Ge}_{\mathrm{x}} \mathrm{Bi}_{\mathrm{y}} \mathrm{Se}_{\mathrm{z}}{ }^{+}$clusters $\left(\mathrm{Ge}_{2} \mathrm{BiSe}_{4} \mathrm{H}^{+}\right.$and $\mathrm{Ge}_{2} \mathrm{Bi}_{2} \mathrm{Se}_{3} \mathrm{H}^{+}$) were also detected. LA also generated several oxygenated bismuth and bismuth selenide clusters. Among all the laser ablation generated clusters from the Ge:Bi:Se (5:1:1) precursor, $\mathrm{Se}_{5}{ }^{+}$and $\mathrm{Bi}^{+}$are the highest intensity peaks, even though the elemental mixture contains an excess of germanium. Surprisingly, the laser ablation synthesis generated a high number of $\mathrm{Bi}_{\mathrm{y}} \mathrm{Se}_{\mathrm{z}}{ }^{+}$clusters rather than $\mathrm{Ge}_{\mathrm{x}}{ }^{+}$clusters. This might indicate that the ionization efficiency of germanium is lower compared to Bi and Se. The mass spectra of Ge:Bi:Se elemental mixtures dispersed in parafilm were also measured, however no significant difference in comparison with those without parafilm were observed.

The mass spectra recorded from Ge:Bi:Se elemental mixtures in the negative ion mode show the formation of a series of clusters such as $\mathrm{Se}_{z}^{-}(z=1-8)$ and $\mathrm{BiSe}_{z}^{-}(z=2-9)$ via laser ablation synthesis. An example of a mass spectrum of Ge:Bi:Se (1:1:5) precursor is given in Fig. 5. The $\mathrm{Se}_{3}{ }^{-}$cluster represents the highest intensity peak in the mass spectra of all the precursors. However, no $\mathrm{Ge}_{x} \mathrm{Se}_{z}{ }^{-}$and $\mathrm{Ge}_{x} \mathrm{Bi}_{y}{ }^{-}$clusters were discovered. Especially in negative ion mode, the $\mathrm{Se}_{z}{ }^{-}$clusters are most prominent with the highest intensity - higher than other binary $\mathrm{Bi}_{y} \mathrm{Se}_{z}^{-}$clusters - which indicate that selenium ionization is more efficient than $\mathrm{Bi}$ and $\mathrm{Ge}$. The mass spectra were 


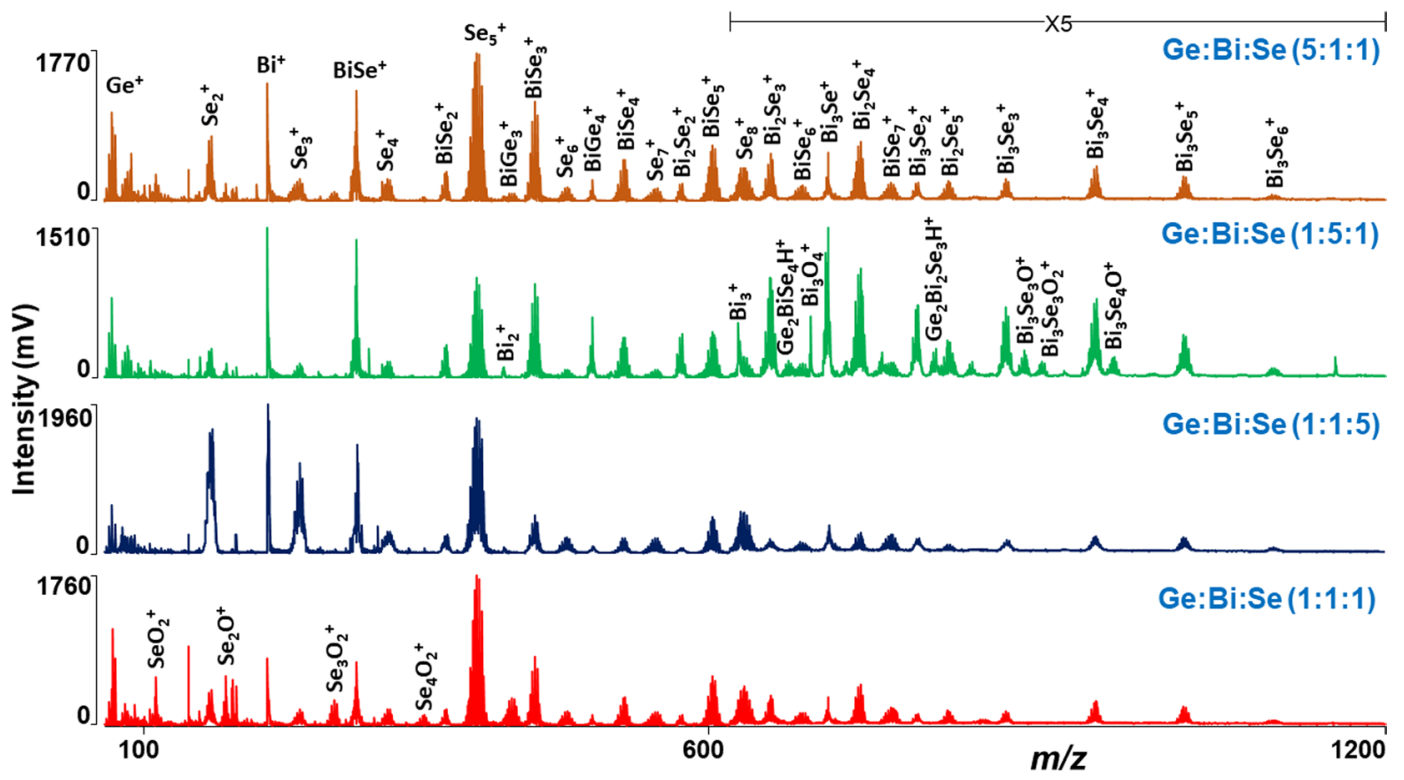

Figure 4. Mass spectra obtained from Ge:Bi:Se elemental mixtures. Conditions: Positive ion mode, laser energy 120 a.u. For the sake of clarity, a detailed description of most of the peaks of Ge:Bi:Se 5:1:1 precursor is given.

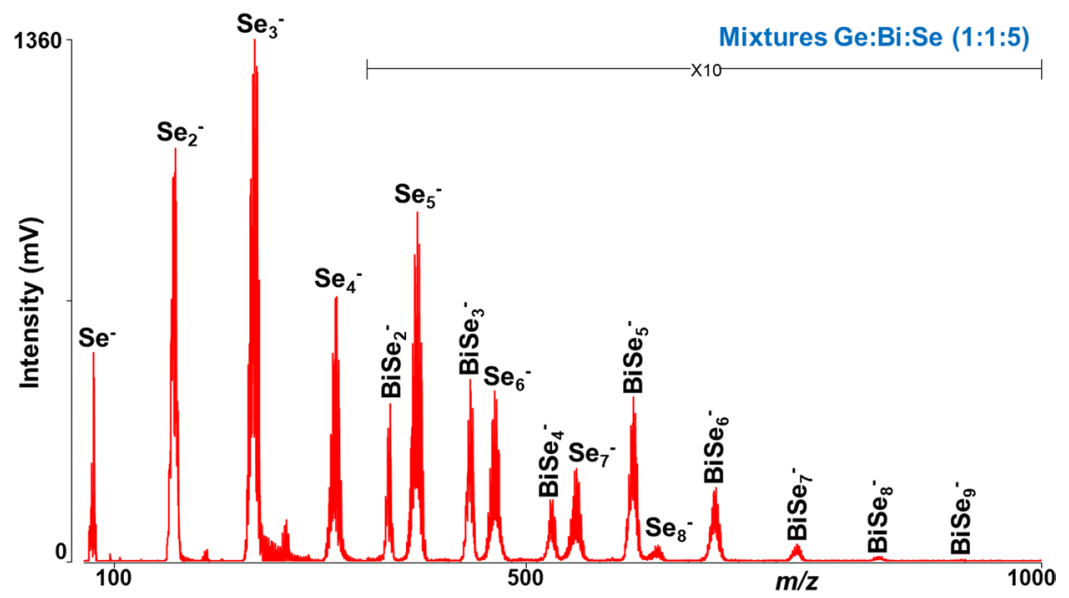

Figure 5. Mass spectrum of Ge:Bi:Se (1:1:5) elemental mixture. Conditions: Negative ion mode, laser energy 110 a.u.

also measured from Ge:Bi:Se elemental mixtures dispersed in parafilm; however no significant difference in the mass spectra were observed, therefore those mass spectra are not shown.

From the results obtained via LA TOFMS of thin films and elemental mixtures, it was deduced that the threshold energy for the thin films was higher than for the elemental mixtures. Also, the number of clusters generated during the laser ablation of elemental mixtures is higher than for the thin films. An explanation for this may be that during the laser ablation of the thin films, generally, the clusters and/or ions formed may result from the original structure of solid material via its fragmentation. This process might need larger laser energy, and therefore high threshold energy was required. In contrast, in the case of the elemental mixtures, the clusters and/or ions are formed via the laser ablation synthesis of generated ion in the plasma plume, which probably needs low laser power, and hence lower threshold energy is needed.

It is worth noting that in all the recorded mass spectra from thin films and elemental mixtures, few $\mathrm{Ge}_{x}^{+/-}$ clusters/ions were detected. As discussed earlier, this might be due to lower ionization of Ge when compared to $\mathrm{Bi}$ or Se. The results are qualitatively comparable to our previously published results, where we observed the same situation with the ionization of germanium when it is present in combination with one or more other element $s^{27,30}$. In order to make a comparison, we have investigated elemental germanium via LA TOFMS under similar experimental conditions. The laser ablation of Ge shows the formation of positively and negatively charged $\mathrm{Ge}_{x}{ }^{+-}$clusters/ions with a maximum of 10 germanium atoms $\left(\mathrm{Ge}_{10}{ }^{+-}\right.$) (the mass spectra are not shown). However, when $\mathrm{Ge}$ is present in the binary (e.g. Ge-Se) or ternary (e.g. Ge-Bi-Se) systems, the pure Ge clusters 

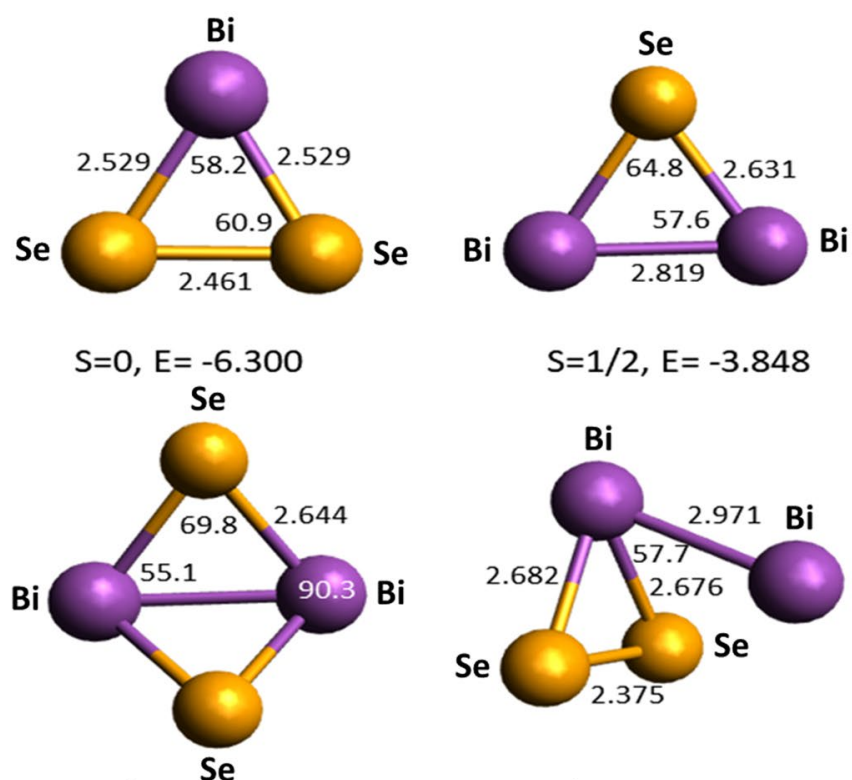

$S=1 / 2, E=-31.041$

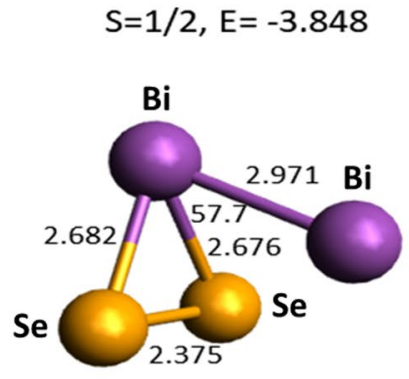

$S=1 / 2, E=-12.787$
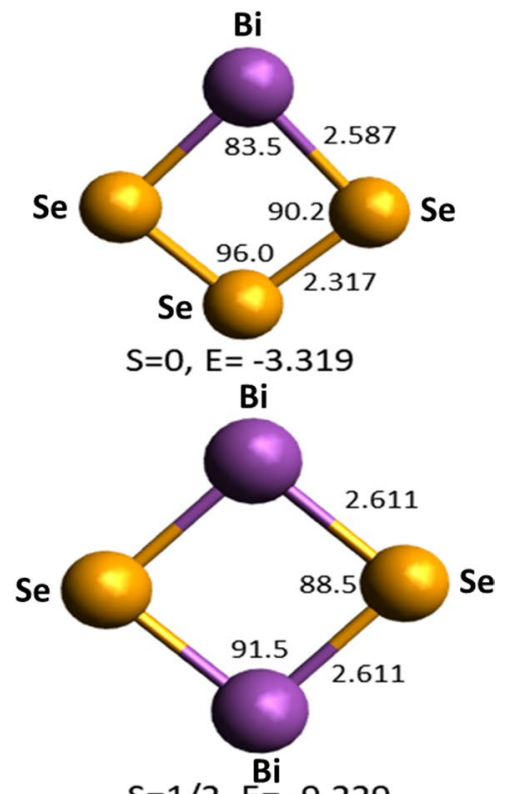

$\mathrm{S}=1 / 2, \mathrm{E}=-9.229$

Figure 6. The optimized structures of selected three- and four-atom clusters of the Bi-Se system. The bond length is given in $\AA$, bond angle in degree, energy (E) in $\mathrm{kcal} / \mathrm{mol}$, and S stands for the spin. The purple and yellow colored spheres represent bismuth and selenium atoms, respectively.

are not seen (except $\mathrm{Ge}_{8}{ }^{+}$and $\mathrm{Ge}^{+}$, cf. Figs. 1 and 2), as $\mathrm{Ge}$ atoms are strongly bound by Se or Bi. For example, the total number of germanium-containing clusters demonstrated in Fig. 2 is equal to 6.

Also, it is noted that the formation of $\mathrm{Ge}_{x} \mathrm{Bi}_{y}{ }^{+-}$clusters is marginal: only two $\left(\mathrm{Ge}_{3} \mathrm{Bi}^{+}\right.$and $\left.\mathrm{Ge}_{4} \mathrm{Bi}^{+}\right)$cationic species were identified in mass spectra of the Ge:Bi:Se (5:1:1) elemental mixture when the content of Ge was high. To understand the process, we examined the elemental mixture (Ge:Bi, 1:1) via LA TOFMS. Surprisingly, no $\mathrm{Ge}_{x} \mathrm{Bi}_{y}{ }^{+-}$clusters were detected. However, it is difficult to judge why those species were not generated.

When looking at LA TOFMS data presented in this work, the results might evidence at least some of the structural units identified from Raman scattering spectroscopy data as reported in [20]. For example, observed $\mathrm{GeSe}_{4}{ }^{+-}$clusters confirm the presence of $\mathrm{GeSe}_{4}$ tetrahedral entities in the structure of co-sputtered amorphous films. On the other hand, most of the detected species seem not to correspond with local structural units evidenced via Raman spectroscopy. The clusters generated might be formed either due to the deeper fragmentation of the original amorphous structure, or some high mass species that are not considered as part of the original amorphous structure can be formed from the highly energetic ions present in the plasma plume, i.e. by laser ablation synthesis.

Selected $G e_{x} S e_{z}, B i_{y} S e_{z}$, and $G e_{x} B i_{y} S e_{z}$ clusters structure optimization. In the past two decades, the atomic and molecular clusters were extensively studied by theoretical chemists to explore their structural and electronic properties. For example, the structures of $\mathrm{Ge}_{x}{ }^{+/-}, \mathrm{Bi}_{y}{ }^{+/-}$, and $\mathrm{Se}_{z}^{+/-}$clusters have already been reported ${ }^{31-35}$. In our previous work, we reported on the formation of about 50 and 33 clusters during the laser ablation mass spectrometric study of $\mathrm{Ge}-\mathrm{Se}^{27}$ and $\mathrm{Bi}-\mathrm{Se}^{36}$ materials. However, the detailed study of $\mathrm{Ge}, \mathrm{Bi}$, and Se binary and ternary combinations clusters' structure has not yet been reported. In this study we carried out a laser ablation mass spectrometric study of the ternary Ge-Bi-Se system; in this way, many binary $\mathrm{Ge}_{x} \mathrm{Se}_{z}^{+/-}, \mathrm{Bi}_{y} \mathrm{Se}_{z}^{+/-}$, and ternary $\mathrm{Ge}_{x} \mathrm{Bi}_{y} \mathrm{Se}_{z}{ }^{+l-}$ clusters are produced and identified. Therefore, the structural properties of selected $\mathrm{Ge}_{x} \mathrm{Se}_{z}^{+l-}$, $\mathrm{Bi}_{y} \mathrm{Se}_{z}^{+l-}$, and $\mathrm{Ge}_{x} \mathrm{Bi}_{y} \mathrm{Se}_{z}^{+/-}$clusters were investigated via DFT calculations. Because this paper is essentially focused on the mass spectrometric investigation of Ge-Bi-Se materials, only a few simple clusters out of many produced species are selected for the structural optimization. Also, it is well known that the number of possible structural isomers increases drastically with the growing number of atoms in the clusters.

Different possible isomers of each selected cluster have been studied and the ground state geometries of each cluster with local minima in the potential energy surface (PES) and lowest energy have been determined (Figs. 68). The geometry optimization has been performed at the PBE0/TZ2 $\mathrm{P}^{37,38}$ level of theory using Gaussian software $^{39}$. For an open-shell system with unpaired electrons, an unrestricted approach was used. To ensure that the calculated geometries correspond to the ground state with local minima in the PES, frequencies were computed at the same level of theory. Absence of imaginary frequency confirms that our structures are indeed in the ground state.

The number of the most stable isomers of three- and four-atom binary $\mathrm{Bi}_{y} \mathrm{Se}_{z}{ }^{+}$clusters $\left(\mathrm{BiSe}_{2}{ }^{+}, \mathrm{Bi}_{2} \mathrm{Se}^{+}, \mathrm{BiSe}_{3}{ }^{+}\right.$, and $\mathrm{Bi}_{2} \mathrm{Se}_{2}{ }^{+}$) are given in Fig. 6. The distance between $\mathrm{Bi}-\mathrm{Bi}$ and Se-Se near-neighbours is found in the range 2.819-2.971 and 2.317-2.461 $\AA$, respectively, while for the heteroatomic Bi-Se, bond length is 2.529-2.682 $\AA$.

Similarly, the optimization of some selected three- and four-atom $\mathrm{Ge}_{x} \mathrm{Se}_{z}{ }^{+}$clusters such as $\mathrm{GeSe}_{2}{ }^{+}, \mathrm{Ge}_{2} \mathrm{Se}_{2}{ }^{+}$, and $\mathrm{GeSe}_{3}{ }^{+}$was performed. The optimized structures are shown in Fig. 7. For each cluster, several isomers were 

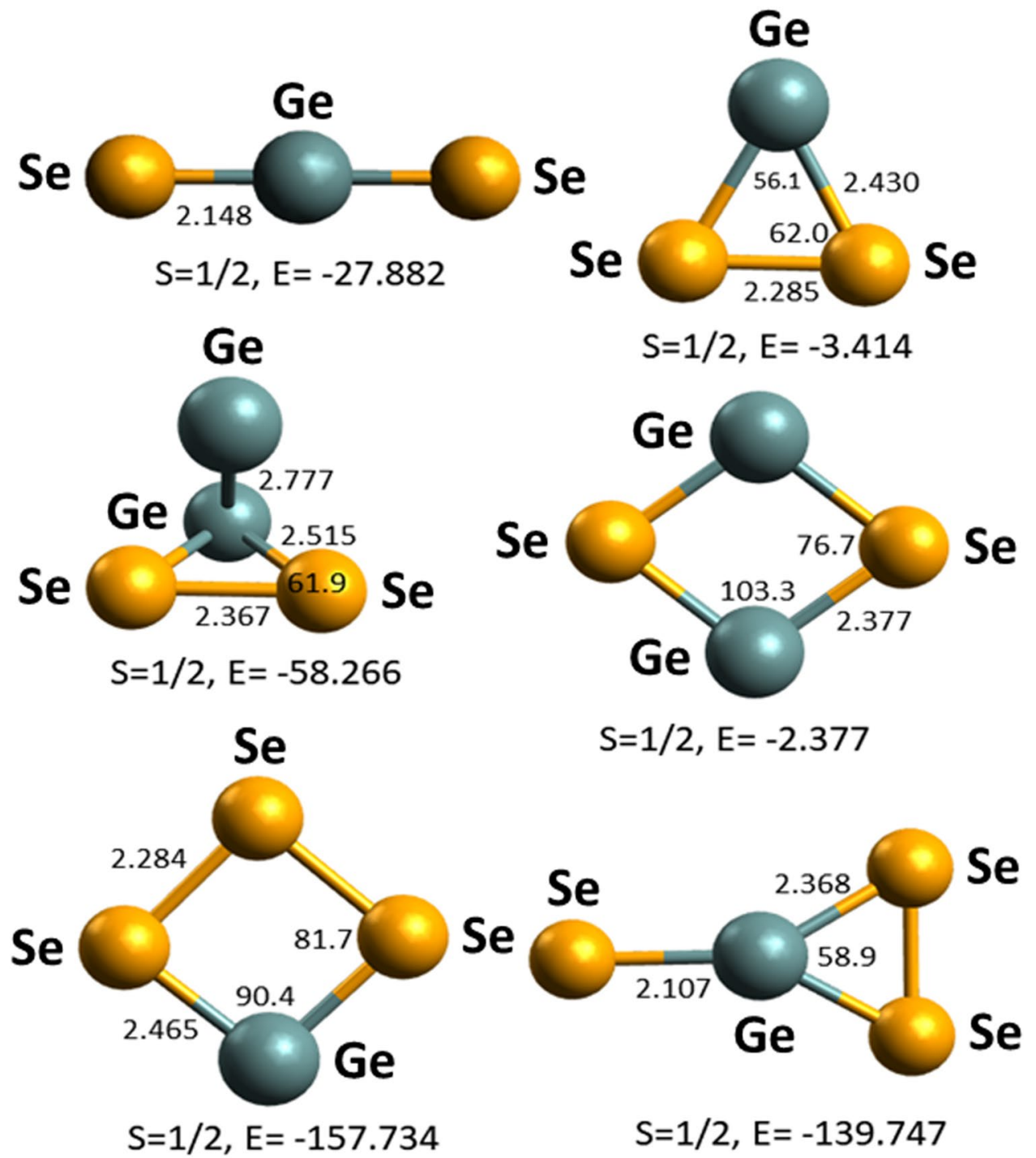

Figure 7. The optimized structures of three- and four-atom clusters of the Ge-Se system. The bond length is given in $\AA$, bond angle in degree, energy (E) in $\mathrm{kcal} / \mathrm{mol}$, and $\mathrm{S}$ stands for spin. The green and yellow colored spheres represent germanium and selenium atoms, respectively.

considered for optimization, but only ground state geometries with the lowest energy are shown in Fig. 7. The bond distance between Ge-Ge, Se-Se, and Ge-Se nuclei were found as 2.777, 2.284-2.367, and 2.107-2.515 , respectively.

Finally, the $\mathrm{GeBiSe}_{3}$ ternary cluster was selected for the structure optimization procedure. The cluster structures were optimized in $+1,0$, and -1 charge. Figure 8 shows the optimized structures of the $\mathrm{GeBiSe}_{3}$ cluster, the last three geometries represent monocationic, neutral and monoanionic isomers. From the calculated data it was observed that the structural parameters such as bond length, bond angle and the geometry of monocationic, neutral and monoanionic structures do not alter much with the charge. The bond length between two neighbouring nuclei Se-Se, Ge-Se, Bi-Se, and Bi-Ge is in the range 2.306-2.434, 2.317-2.676, 2.571-2.712, and 2.593-2.923 respectively.

\section{Conclusions}

LA with TOFMS analysis of thin films as well as elemental mixtures of Ge-Bi-Se system produces many unary, binary and some ternary clusters. The thin films were prepared via rf magnetron co-sputtering using $\mathrm{GeSe}_{2}$ and $\mathrm{Bi}_{2} \mathrm{Se}_{3}$ targets. Six different compositions of thin films were examined, each one producing about 20 different positively and negatively charged clusters. These might be considered as fragments of local structure of studied materials that are present in plasma during thin films deposition. On the other hand, in the case of the elemental mixtures, a higher number (about 28) of clusters were generated at lower laser energy. Finally, structures of some selected binary and ternary clusters were calculated using DFT optimization.

In conclusion, laser ablation time-of-flight mass spectrometry is considered as a useful analytical technique to study amorphous chalcogenide thin films in terms of identification of species present in the plasma phase when 

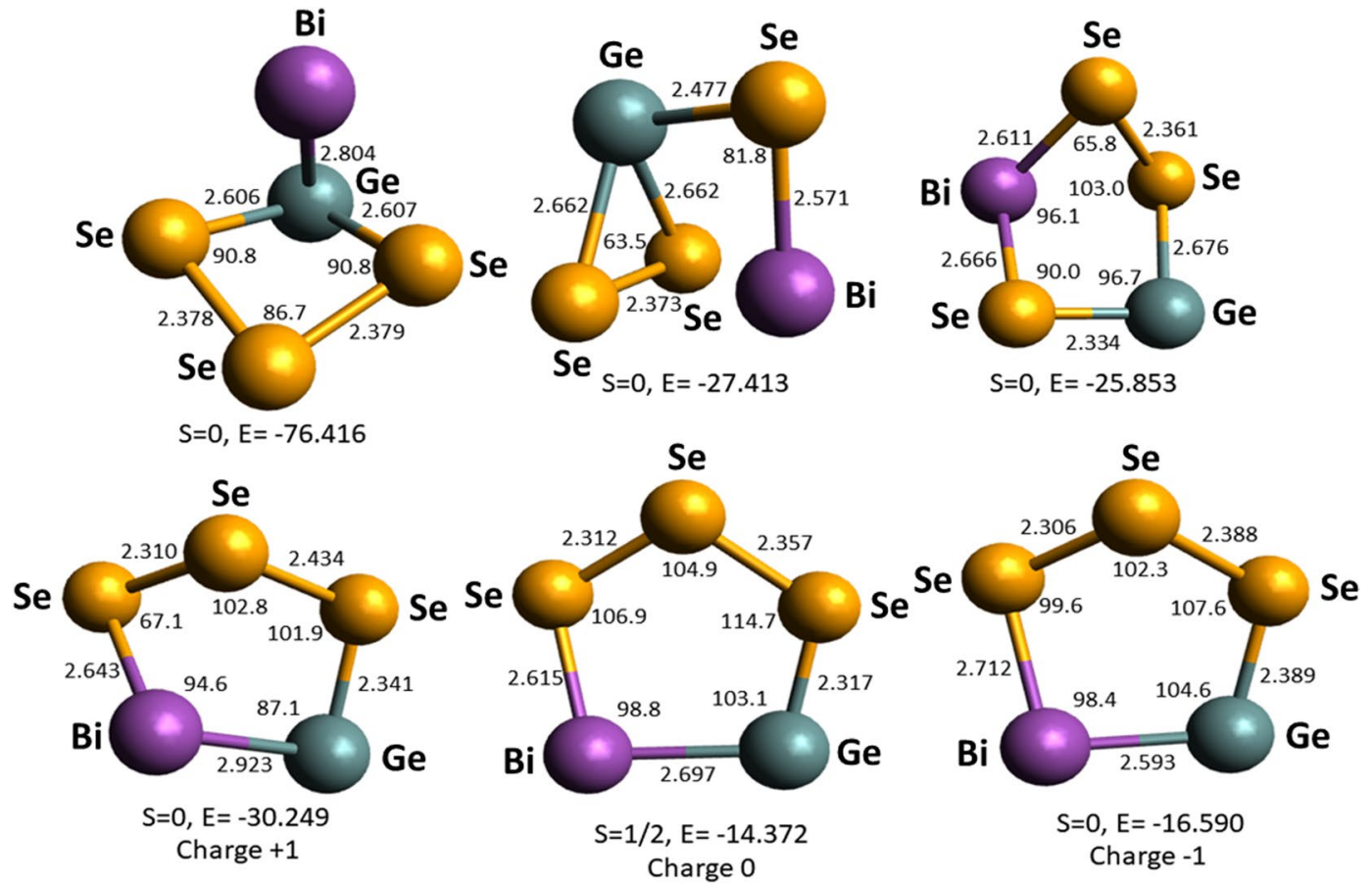

$S=0, E=-16.590$

Charge -1

Figure 8. The optimized structural isomers of the $\mathrm{GeBiSe}_{3}$ cluster. The last three geometries represent monocationic, neutral, and monoanionic structures. The bond length is given in $\AA$, bond angle in degree, energy (E) in $\mathrm{kcal} / \mathrm{mol}$ and $\mathrm{S}$ stands for spin. The purple, green, and yellow colored spheres represent bismuth, germanium and selenium, respectively.

the material is exposed to laser pulses. The knowledge of stoichiometry of the species might help to obtain partial structural information of the thin films. Laser ablation of thin films and knowledge of plasma based on TOFMS analysis can also be generally helpful in understanding specific industrial processes such as laser surface treatment and laser additive manufacturing.

\section{Materials and Methods}

Chemicals. Water used was double distilled from a quartz apparatus Heraeus Quarzschmelze (Hanau, Germany). Germanium, bismuth, selenium ( $5 \mathrm{~N}$ purity), acetone, methanol and acetonitrile were purchased from Sigma-Aldrich (Steinheim, Germany). Red phosphorus was bought from Riedel de Haën (Hannover, Germany). Micro-90 (cleaning agent) was from Kratos (Manchester, UK). Xylene (a mixture of isomers) was purchased from Mikrochem Spol. s.r.o. (Pezinok, Slovak Republic) and parafilm from Pechiney Plastic Packaging (Chicago, IL, USA). Silicon wafers used as substrates for the thin films deposition were purchased from ON Semiconductor (Czech Republic). All other reagents were of analytical grade purity.

Preparation of thin films. Amorphous Ge-Bi-Se thin films were deposited using conventional $\mathrm{rf}$ $(13.56 \mathrm{MHz})$ magnetron sputtering technique. The experimental conditions of the deposition process are described elsewhere ${ }^{23}$. For the deposition of the Ge-Bi-Se thin films, commercial polycrystalline $\mathrm{GeSe}_{2}$ and $\mathrm{Bi}_{2} \mathrm{Se}_{3}$ sputtering targets (ALB Materials, Inc., Henderson, NV, USA) were used.

Mixtures of $\mathrm{Ge}, \mathrm{Bi}$, and Se. Appropriate amounts of high purity germanium, bismuth, and selenium elements were mixed together in an agate mortar to get the desired composition $(1: 1: 1,1: 1: 5,1: 5: 1$, and 5:1:1 molar ratios). The mixture was then suspended in acetonitrile applying ultrasonication for $1 \mathrm{~min}$ and finally used for mass spectrometric measurements.

About $2 \mathrm{mg}$ of each elemental mixture was dispersed separately in parafilm solution prepared by dissolving a piece of $(1 \mathrm{~cm} \times 1 \mathrm{~cm})$ parafilm in xylene $(2 \mathrm{ml})$ using ultrasonication, and then used further for the experiments.

Instrumentation. The surface morphology and chemical composition of the thin films were determined by using a scanning electron microscope (SEM) with an energy dispersive X-ray analyzer (EDS, TESCAN VEGA 3 EasyProbe). The EDS measurements were performed at 3 different spots on the thin films for each sample and averaged. The uncertainty of EDS measurements for the studied films is \pm 1 at. \%. The X-ray diffraction (XRD) technique (D8-Advance diffractometer, Bruker AXS, Germany) was employed to confirm the amorphous state of co-sputtered thin films. The diffraction angles were measured at room temperature from 5 to $65^{\circ}(2 \theta)$ within $0.02^{\circ}$ steps. 
The target plate was cleaned according to the Shimadzu target cleaning procedure. Specifically, it includes cleaning with water followed by ultrasonic cleaning separately in Micro-90 solution, acetone, and methanol for $15 \mathrm{~min}$ and finally rinsing several times with water and acetonitrile.

$1 \mu \mathrm{L}$ of acetonitrile suspension of the sample powder $(1 \mathrm{mg} / \mathrm{mL})$ was deposited on the target plate and dried in a stream of air at room temperature and LA TOF mass spectra were recorded. Thin films were fixed on the target plate using adhesive tape; a small portion of the thin films was coated manually with a parafilm solution and then measured.

An AXIMA CFR mass spectrometer from Kratos Analytical Ltd. (Manchester, UK) equipped with a nitrogen laser $(337 \mathrm{~nm})$ and a time-of-flight mass analyzer was used to record the mass spectra. The laser repetition rate was set to $5 \mathrm{~Hz}$ with a pulse duration of $3 \mathrm{~ns}$. The exposed sample spot size was approximately $150 \mu \mathrm{m}$ in diameter, giving the laser energy fluence $\sim 1 \mathrm{~J} \mathrm{~cm}^{-2}$.

Mass spectra were recorded in both positive and negative ion modes by accumulating the data from about 1000 laser shots. The laser energy varied in arbitrary units (a. u.) from 0 to 180; this relative scale is used hereafter. External calibration was done using red phosphorus clusters, while the accuracy achieved was below $\pm 50 \mathrm{mDa}$.

Software and computation. Theoretical isotopic patterns were calculated using Launchpad software (Kompact version 2.9.3, 2011) from Kratos Analytical Ltd. (Manchester, UK). The calculated structures were visualized using Avogadro: an open-source molecular builder and visualization tool (version 1.2.0., http://avogadro.cc//) ${ }^{40}$.

\section{Data availability}

The datasets generated and analyzed during the current study are available from the corresponding author on reasonable request.

Received: 7 October 2019; Accepted: 3 December 2019;

Published online: 16 December 2019

\section{References}

1. Adam, J.-L. \& Zhang, X. Chalcogenide glasses: Preparation, properties and applications. (Woodhead publishing limited (UK), 2014).

2. Eggleton, B. J., Luther-Davies, B. \& Richardson, K. Chalcogenide photonics. Nat. Photonics 5, 141-148 (2011).

3. Soonmin, H. \& Joseph Sahaya Anand, T. A. Review of chalcogenide thin films for solar cell applications. Indian J. Sci. Technol. 8, $67499(2015)$

4. Wuttig, M., Bhaskaran, H. \& Taubner, T. Phase-change materials for non-volatile photonic applications. Nat. Photonics 11, 465-476 (2017).

5. Raoux, S., Wełnic, W. \& Lelmini, D. Phase change materials and their application to nonvolatile memories. Chem. Rev. 110, 240-267 (2010).

6. Shiryaev, V. S. et al. Preparation of core-clad arsenic rich As-Se glass fiber. J. Non. Cryst. Solids 448, 11-15 (2016).

7. Zakery, A. \& Elliott, S. R. Optical properties and applications of chalcogenide glasses: a review. J. Non. Cryst. Solids 330, 1-12 (2003).

8. Němec, P. et al. Optical properties of $\left(\mathrm{GeSe}_{2}\right)_{100-x}\left(\mathrm{Sb}_{2} \mathrm{Se}_{3}\right)_{x}$ glasses in near- and middle-infrared spectral regions. Mater. Res. Bull. 51, 176-179 (2014)

9. Sourková, P. et al. Spectroscopy of infrared transitions of $\mathrm{Pr}^{3+}$ ions in Ga-Ge-Sb-Se glasses. J. Lumin. 129, 1148-1153 (2009).

10. Afify, N. Structural relaxation of $\mathrm{GeSe}_{2}$ chalcogenide glass studied with use of the radial distribution function. Phys. Rev. B 48, 16304-16309 (1993).

11. Popescu, M. Non-crystalline chalcogenides. (Kluwer Academic Publishers, 2000).

12. Borisova, Z. U. Glassy Semiconductors. (Springer US, 1981).

13. Tohge, N., Minami, T. \& Tanaka, M. Photoconductivity of vitreous chalcogenides chemically modified by bismuth. J. Non. Cryst. Solids 59-60, 999-1002 (1983).

14. Tohge, N., Yamamoto, Y., Minami, T. \& Tanaka, M. Preparation of n-type semiconducting $\mathrm{Ge}_{20} \mathrm{Bi}_{10} \mathrm{Se}_{70}$ glass. Appl. Phys. Lett. 34, 640-641 (1979).

15. Frumar, M. \& Tichý, L. N-type conductivity in chalcogenide glasses and layers. J. Non. Cryst. Solids 97-98, 1139-1146 (1987).

16. Nagels, P., Rotti, M. \& Vikhrov, S. Doping of chalcogenide glasses in the Ge-Se and Ge-Te systems. J. Phys. Colloq. C4, 907 (1981).

17. Abdel-Rahim, F. M., Hafiz, M. M. \& Alsorory, H. Structure and electrical properties of $\mathrm{Bi}_{5} \mathrm{Ge}_{x} \mathrm{Se}_{95-x}$ thin films. Curr. Appl. Phys. 12, 389-393 (2012)

18. Aparimita, A. et al. Effect of Bi addition on the optical properties of $\mathrm{Ge}_{30} \mathrm{Se}_{70-x} \mathrm{Bi}_{x}$ thin films. J. Alloys Compd. 739, 997-1004 (2018).

19. Liu, Y. et al. A study on crystallization kinetics of thermoelectric $\mathrm{Bi}_{2} \mathrm{Se}_{3}$ crystals in $\mathrm{Ge}-\mathrm{Se}-\mathrm{Bi}$ chalcogenide glasses by differential scanning calorimeter. J. Am. Ceram. Soc. 96, 2141-2146 (2013).

20. Mytilineou, E., Chao, B. S. \& Papadimitriou, D. Raman scattering in sputtered amorphous $\mathrm{Ge}_{25} \mathrm{Se}_{75-x} \mathrm{Bi}_{x}$ films. J. Non. Cryst. Solids 195, 279-285 (1996).

21. Kounavis, P. \& Mytilineou, E. The defect states in sputtered Ge-Se-Bi films. J. Non. Cryst. Solids 201, 119-127 (1996).

22. Springer Handbook of Glass. (eds. Musgraves, J. D., Hu, J. \& Calvez, L.) (Springer International Publishing, 2019).

23. Halenkovič, T. et al. Amorphous Ge-Sb-Se thin films fabricated by co-sputtering: Properties and photosensitivity. J. Am. Ceram. Soc. 101, 2877-2887 (2018).

24. Mawale, R. et al. Mass spectrometric investigation of amorphous Ga-Sb-Se thin films. Sci. Rep. 9, 10213 (2019),

25. Šútorová, K. et al. Laser desorption ionisation time-of-flight mass spectrometry of chalcogenide glasses from $\left(\mathrm{GeSe}_{2}\right)_{100-x}\left(\mathrm{Sb}_{2} \mathrm{Se}_{3}\right)_{x}$ system. J. Am. Ceram. Soc. 98, 4107-4110 (2015).

26. Mawale, R. M. et al. Laser desorption ionization of $\mathrm{As}_{2} \mathrm{Ch}_{3}(\mathrm{Ch}=\mathrm{S}$, Se, and Te) chalcogenides using quadrupole ion trap time-offlight mass spectrometry: A comparative study. J. Am. Soc. Mass Spectrom. 28, 2569-2579 (2017).

27. Mawale, R. et al. Laser desorption ionization time-of-flight mass spectrometry of $\mathrm{Ge}_{x} \mathrm{Se}_{1-x}$ chalcogenide glasses, their thin films, and Ge:Se mixtures. J. Non. Cryst. Solids 509, 65-73 (2019).

28. Rienstra-Kiracofe, J. C., Tschumper, G. S., Schaefer, H. F., Nandi, S. \& Ellison, G. B. Atomic and molecular electron affinities: Photoelectron experiments and theoretical computations. Chem. Rev. 102, 231-282 (2002).

29. Kramida, A., Ralchenko, Y., Reader, J. \& Team, N. A. NIST Atomic Spectra Database (version 5.6.1). Natl. Inst. Stand. Technol. Gaithersburg, MD., https://doi.org/10.18434/T4W30F (2018).

30. Pangavhane, S. D. et al. Laser desorption ionization time-of-flight mass spectrometry of erbium-doped Ga-Ge-Sb-S glasses. Rapid Commun. Mass Spectrom. 28, 1221-1232 (2014). 
31. Sharma, T., Sharma, R., Tamboli, R. A. \& Kanhere, D. G. Ab initio investigation of structural and electronic properties of selenium and tellurium clusters. Eur. Phys. J. B 92 (2019).

32. Archibong, E. F. \& St-Amant, A. A study of $\mathrm{Ge}_{n}{ }^{-}$and $\mathrm{Ge}_{n}(n=2-6)$ using B3LYP-DFT and CCSD(T) methods: The structures and electron affinities of small germanium clusters. J. Chem. Phys. 109, 962-972 (1998).

33. Jia, J. M., Chen, G. B., Shi, D. N. \& Wang, B. L. Structural and electronic properties of $\mathrm{Bi}_{n}(n=2-14)$ clusters from density-functional calculations. Eur. Phys. J. D 47, 359-365 (2008).

34. Yuan, H. K., Chen, H., Kuang, A. L., Miao, Y. \& Xiong, Z. H. Density-functional study of small neutral and cationic bismuth clusters $\mathrm{Bi}_{n}$ and $\mathrm{Bi}_{n}{ }^{+}(n=2-24) . J$. Chem. Phys. 128, 094305 (2008).

35. Xu, W. \& Bai, W. The selenium clusters $\mathrm{Se}_{n}(n=1-5)$ and their anions: Structures and electron affinities. J. Mol. Struct. THEOCHEM 854, 89-105 (2008).

36. Mandal, G., Mawale, R. \& Havel, J. Laser Ablation Generation of Bismuth Selenide Clusters from Mixtures of Elements, Crystalline $\mathrm{Bi}_{2} \mathrm{Se}_{3}$ or Thin Films: Laser Desorption Ionization (LDI) and Surface Assisted LDI (SALDI) Time-of-Flight Mass Spectrometry using Graphene and Nano- diamonds. (In Press) (2019).

37. van Lenthe, E. \& Baerends, E. J. Optimized Slater-type basis sets for the elements 1-118. J. Comput. Chem. 24, 1142-1156 (2003).

38. Swart, M., Ehlers, A. W. \& Lammertsma, K. Performance of the OPBE exchange-correlation functional. Mol. Phys. 102, 2467-2474 (2004).

39. Frisch, M. J. et al. Gaussian 16, Revision C.01. Gaussian, Inc., (Wallingford CT, 2016).

40. Hanwell, M. D. et al. Avogadro: An advanced semantic chemical editor, visualization, and analysis platform. J. Cheminform. 4, 17 (2012).

\section{Acknowledgements}

The authors are grateful to the Czech Science Foundation (Project No. 18-03823S) for supporting this work.

\section{Author contributions}

P.N., J.H. and V.N. planned and managed the project. P.N. designed this work, J.G. prepared the thin films, M.B. performed the characterization of thin films and analyzed the data with the help of P.N. and V.N. R.M. examined the thin films with LA TOFMS and analyzed the data with the help of P.N. and J.H. The manuscript was written by R.M. with the help of P.N. and J.H. Ge-Bi-Se mixtures were prepared and examined by G.M. The structure of the clusters was computed by R.M. with the help of P.L.B.

\section{Competing interests}

The authors declare no competing interests.

\section{Additional information}

Correspondence and requests for materials should be addressed to R.M. or P.N.

Reprints and permissions information is available at www.nature.com/reprints.

Publisher's note Springer Nature remains neutral with regard to jurisdictional claims in published maps and institutional affiliations.

(c) (1) Open Access This article is licensed under a Creative Commons Attribution 4.0 International License, which permits use, sharing, adaptation, distribution and reproduction in any medium or format, as long as you give appropriate credit to the original author(s) and the source, provide a link to the Creative Commons license, and indicate if changes were made. The images or other third party material in this article are included in the article's Creative Commons license, unless indicated otherwise in a credit line to the material. If material is not included in the article's Creative Commons license and your intended use is not permitted by statutory regulation or exceeds the permitted use, you will need to obtain permission directly from the copyright holder. To view a copy of this license, visit http://creativecommons.org/licenses/by/4.0/.

(c) The Author(s) 2019 